\title{
Article \\ A 560 GHz Sub-Harmonic Mixer Using Half-Global Design Method
}

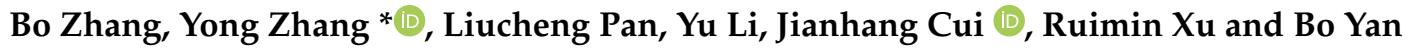

School of Electronic Science and Engineering, University of Electronic Science and Technology of China, Chengdu 611731, China; 201921021010@std.uestc.edu.cn (B.Z.); 201921021012@std.uestc.edu.cn (L.P.); 201821020216@std.uestc.edu.cn (Y.L.); cuijianhangdior@163.com (J.C.); rmxu@uestc.edu.cn (R.X.); yanbo@uestc.edu.cn (B.Y.)

* Correspondence: yongzhang@uestc.edu.cn

Citation: Zhang, B.; Zhang, Y.; Pan, L.; Li, Y.; Cui, J.; Xu, R.; Yan, B. A 560 $\mathrm{GHz}$ Sub-Harmonic Mixer Using Half-Global Design Method. Electronics 2021, 10, 234. https:// doi.org/10.3390/electronics10030234

Academic Editor:

Hirokazu Kobayashi

Received: 9 November 2020

Accepted: 17 January 2021

Published: 20 January 2021

Publisher's Note: MDPI stays neutral with regard to jurisdictional claims in published maps and institutional affiliations.

Copyright: (c) 2021 by the authors. Licensee MDPI, Basel, Switzerland. This article is an open access article distributed under the terms and conditions of the Creative Commons Attribution (CC BY) license (https:// creativecommons.org/licenses/by/ $4.0 /)$.

\begin{abstract}
In this paper, a $560 \mathrm{GHz}$ terahertz sub-harmonic mixer using a new half-global design method is reported. This method combines the advantages of the subdivision design method and the global design method, and greatly enhances the abilities of the optimization of matching variables while retaining the portability of the unit circuit. When the local oscillator (LO) frequency was fixed with $3 \mathrm{~mW}$ power at $280 \mathrm{GHz}$, average up-conversion double sideband (DSB) conversion loss of $8 \mathrm{~dB}$ with intermediate frequency (IF) power of $-5 \mathrm{dBm}$ was achieved.
\end{abstract}

Keywords: sub-harmonic mixer; half-global design method; Schottky diodes; 3D-EM model; double sideband conversion loss

\section{Introduction}

In recent years, with the rapid development of the semiconductor technology, terahertz circuits operating above $300 \mathrm{GHz}$ have been applied in object imaging, planetary exploration, and atmospheric remote sensing [1-6]. In these applications, the generation and detection of terahertz band signals are critical links. As the main component of terahertz solid-state receivers, the performance and reliability of terahertz mixers are critical to the entire receiving system. Traditionally, according to nonlinear devices, mixers can be divided into four types: hot electron bolometer (HEB) [7], super-conductor insulator superconductor (SIS) [8], transistor mixers [9], and Schottky barrier diode (SBD) mixers [10]. Among these, the Schottky diode mixer is the preferred choice due to its characteristics of working at room temperature, low requirements for working environment, simple circuit structure, more convenient processing for hybrid integrated forms, and good noise performance [11,12]. The circuit topologies used in terahertz Schottky mixers can be categorized into several types including single-ended, single-balanced [13], and double-balanced [14]. However, these types of mixer cannot be applied to the terahertz band due to problems such as high requirements for the local oscillator (LO) source and difficulty in circuit design. In terahertz frequency bands, a more reasonable solution is harmonic mixing. Using the second harmonic generated by diodes, the LO frequency can be lowered by half of the radio frequency $(\mathrm{RF})$, thus improving performance [15].

Generally, the subdivision design method (SDM) is employed to design terahertz Schottky diode mixers [16,17], in which all unit circuits are simulated independently. However, as the operating frequency increases, processing errors and parasitic effects of the SDM arise for smaller shield cavities and complicated stub matching structures. To solve these problems, a novel global design method (GDM) was proposed. This method greatly increases the matching space [18]. However, the GDM also has its own defects, such as too many variables and long optimization time.

In this paper, we apply a half-global design method (HGDM), which was proposed and verified in the design of a 200-240 GHz sub-harmonic mixer [19]. In the current 
study, this design method was applied for a $560 \mathrm{GHz}$ Schottky diode sub-harmonic mixer. At $560 \mathrm{GHz}$, the circuit processing difficulty increases greatly, and the circuit design is required to be simple. Moreover, the circuit substrate is very thin. To ensure a specific aspect ratio of the circuit substrate, the length of the entire circuit needs to be strictly controlled. Thus, in this study, the matching network was carefully designed and optimized using the subdivision design method, and the remaining circuits of the mixer were designed using the global design method. As a result, the optimization time was reduced, and the optimization space and the circuits' portability were improved. Based on this method, a $560 \mathrm{GHz}$ sub-harmonic mixer is presented.

\section{Diode Modeling}

The nonlinear effect of the diode arises from the Schottky junction. Due to the significant impacts of parasitic parameters at high frequency bands [20], the characteristics of the diodes cannot be fully characterized by the SPICE (Simulation Program with Integrated Circuit Emphasis) model in the design of terahertz circuits. The impacts caused by parasitic parameters must be accurately described. Therefore, the combination of the SPICE model and the three-dimensional (3D) electromagnetic model is employed to represent its nonlinear characterizations. In general, these two models are handled by non-linear simulations, such as the harmonic balance in the Advanced Design System (ADS) and 3-D simulation in the High Frequency Structure Simulator (HFSS) [21].

The SPICE model is a model based on the PN junction (the contact area of Positive type doped area and Negative type doped area) of the diode. It can be equivalent to the nonlinear characteristics of the Schottky diode, but the characteristics of the Schottky diode based on the metal-semiconductor junction cannot be truly reflected. The I-V curve must be simulated and fitted, and then the model should be revised by the comparison with the test curve. The goal of the establishment of a 3D electromagnetic model of the planar SBDs is to achieve an accurate model of the mixer. The model of the anti-parallel SBDs is shown in Figure 1a.

The detailed modeling processes in the HFSS are as follows. Firstly, the structure dimensions of diodes are obtained from the manufacturer, or measured with a sweep electron microscope (SEM) and calculated. Then, the material of each layer is defined, for which the doped buffer layer and epitaxial layer materials must be approximated. To facilitate the establishment of internal ports, the heavily doped buffer layer is set as a perfect conductor (PEC), and the lightly doped epitaxial layer is directly deemed to be GaAs. Finally, the diode anode probe extends through the epitaxial layer to establish the diode internal ports, and two wave ports are set inside the SBDs, as shown in Figure 1b. Note that the same effect can be achieved by setting another wave port at the cathode. The detailed analysis can refer to [22]. In addition, diode assembly should be considered. Thus, the selected transmission lines are included in the construction of an assembly model. Wave ports must be set at both ends of the transmission line. In this way, both the influences of the diodes' parasitic parameters and the diodes' assembly are considered.

After the structure dimensions, material properties and electromagnetic characteristics of the diodes are presented, and the 3D full-wave electro-magnetic model used to describe the high frequency characteristics of the diodes is accurately built. By combining the 3D electromagnetic model with the SPICE model, a complete SBD model is set up. The junction capacitance (Cj0) is $0.95 \mathrm{fF}$, the series resistance (Rs) is $14 \mathrm{ohms}$, the reverse breakdown voltage $(\mathrm{Vj})$ is $0.85 \mathrm{~V}$, and the reverse saturation current (Is) is $0.81 \mathrm{fA}$. The equivalent circuit diagram of the anti-parallel diodes is shown in Figure 1c. 


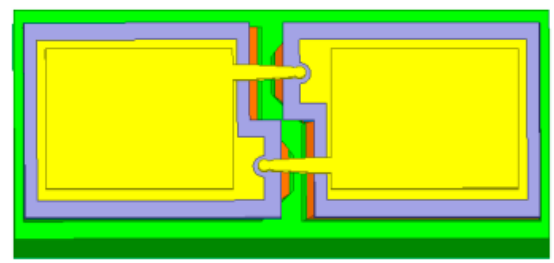

(a)

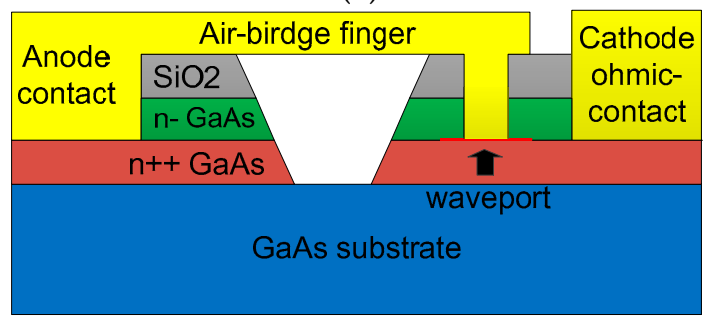

(b)

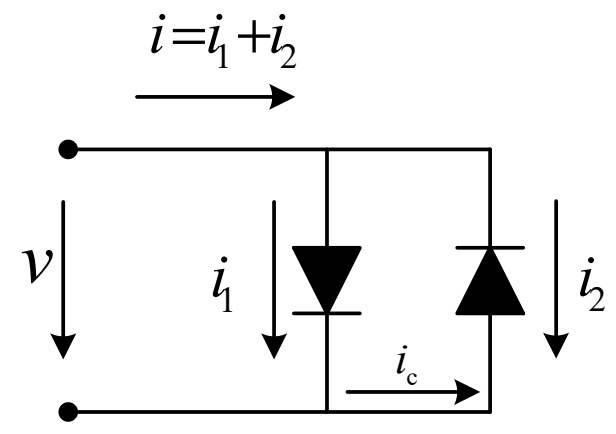

(c)

Figure 1. Planar Schottky barrier diode (SBD) chip AP1-G2-0P64: (a) 3-D electromagnetic model; (b) establishment of Schottky diode wave port; (c) Schematic diagram of anti-parallel diodes equivalent circuit.

\section{Circuit Design}

The mixer was designed using a combination of the ADS and HFSS. In this HGDM, the RF probe and the LO-IF (intermediate frequency) duplexer were designed separately. The suspended micro-strip (SMS), which has a significant impact on circuit matching, was globally optimized. In the SMS, gradient lines and high and low impedance lines are used. The matching and filtering functions between the diodes and the LO probe were realized at the same time, so that the overall length of the SMS was shortened. The model of the terahertz sub-harmonic mixer based on the HGDM is shown in Figure 2. First, to obtain the complete design parameters, the model of diodes was established and its I-V test curve was fitted. Then, the circuit was divided into left and right components for analysis. The left LO duplex was treated as a unit circuit and designed separately. The other unit circuits were split into smaller transmission units and imported to the HFSS for optimization. To realize energy transmission, impedance matching was done between the anti-parallel diodes and the LO, RF port, respectively. All of the unit circuits and diodes model were eventually connected, and an entire circuit model was set up to verify whether it meets the goals. Repeated iterations are required in the restoration process. The remaining transmission lines can be continuously optimized after restoring part of circuits. If the results cannot achieve the specifications, the circuits should be re-optimized. The conversion loss of the mixer and return losses of RF and LO ports are optimization targets. They are finally imported into the ADS for verification. In this way, the portability of some units is preserved, and the optimization space of the circuit is improved. 


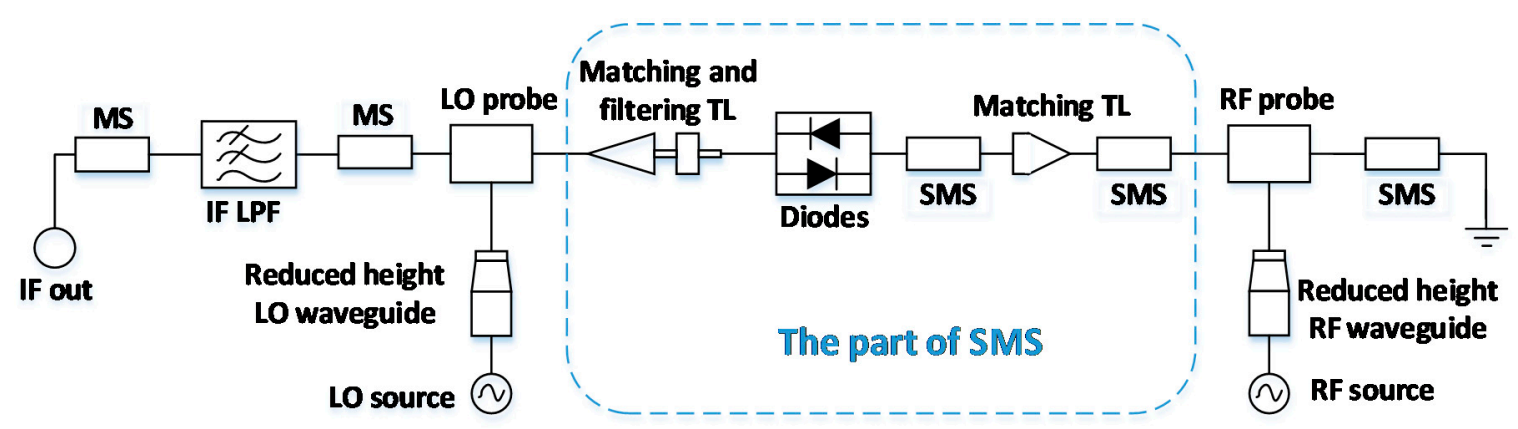

Figure 2. The model of terahertz sub-harmonic mixer based on the half-global design method (HGDM).

The schematic diagram of the split circuit is shown in Figure 3. The anti-parallel SBD Teratech AP1-G2-0P64 was chosen as the key component of the $560 \mathrm{GHz}$ sub-harmonic mixer. A quartz substrate with thickness of 30 um was utilized. A gold conductor with thickness of $4 \mu \mathrm{m}$ was used for the whole mixer circuit. The SMS structure was selected between the LO and RF ports, and the K connector was fixed to the IF output. The size of the channel was determined to be $0.19 \mathrm{~mm}$ wide and $0.12 \mathrm{~mm}$ high. Diodes need to be matched with RF probes and duplexers. The filtering part located between the $\mathrm{LO}$ waveguide and the diodes prevents the RF signal from leaking into the LO port. In addition, a direct current (DC) ground was designed to derive the DC generated by the unbalanced diodes. The LO is input through the standard rectangular waveguide of WR-3 $(0.8636 \mathrm{~mm} \times 0.4318 \mathrm{~mm})$, the RF through the rectangular waveguide of WR-1.9 $(0.483 \mathrm{~mm} \times 0.241 \mathrm{~mm})$, and the IF output in the LO side through a filter which blocks both RF and LO signals. A single-stage reduced technology was applied for the RF/LO waveguide transition and two gold wires were bonded for the IF end of the substrate.

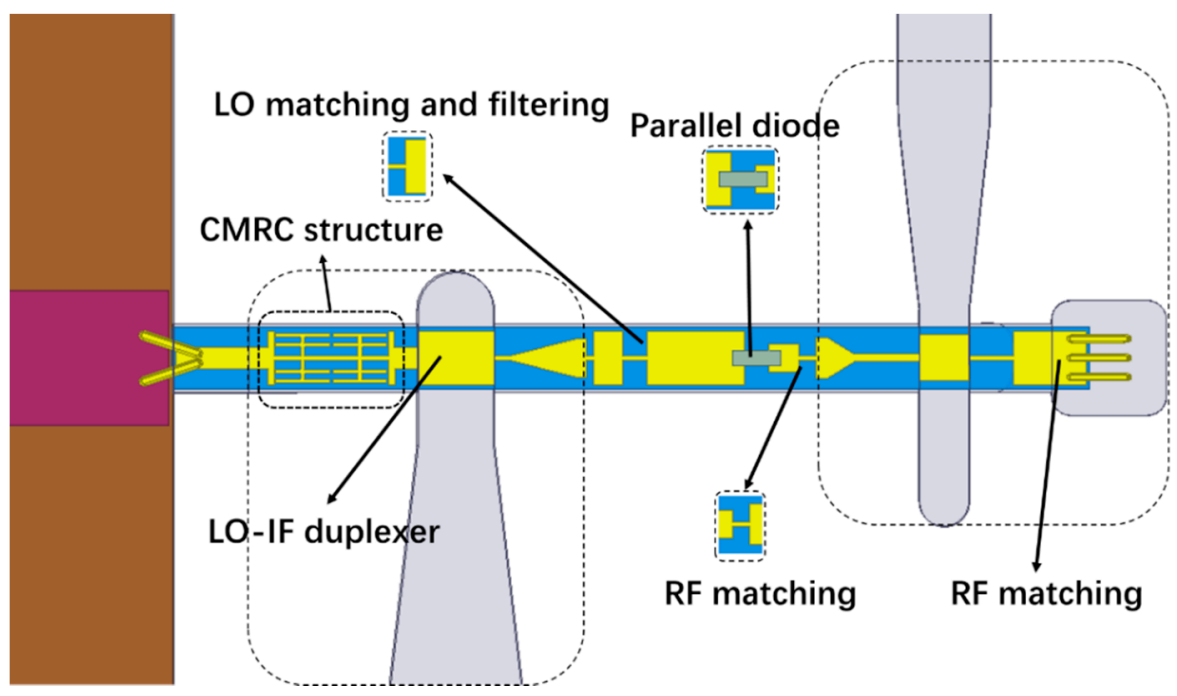

Figure 3. Schematic diagram of the split circuit.

A linear compact microstrip resonant cell (CMRC) structure was employed for the IF low-pass filter, which has good slow wave characteristics. The CMRC is also simple to design and easy to process. Compared with high and low impedance filters, the rectangular coefficient and high-frequency stopband characteristics of the CMRC filter are better, and the length is greatly reduced. Two CMRC units were cascaded to ensure good filtering performance. The total length of the cascaded CMRC was $0.35 \mathrm{~mm}$. In the simulation, the return loss within the passband and the transmission loss outside the passband were both better than $40 \mathrm{~dB}$. The structure of the IF low-pass filter and the final simulation result are shown in Figure 4. 

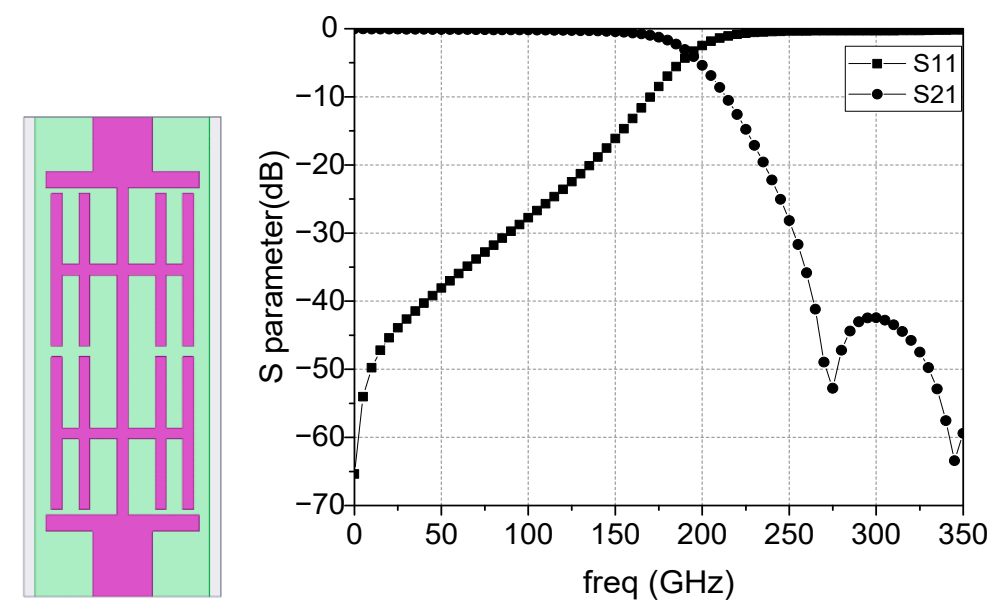

Figure 4. The structure of the low-pass filter and its S-parameter.

It is necessary that the structure of the transition probe has the characteristics of wide frequency band, low loss, low standing wave and ease of processing. The E-plane probe transition structure was selected to couple RF signals due to its lower signal leakage than that of the H-plane probe. The RF signal was input through the reduced-height WR-1.9 waveguide and then transmitted to the SMS. Because of the DC generated by the unbalanced diodes, it was necessary to connect the SMS to the ground via three gold wires. The simulation results show that within the range of $537-587 \mathrm{GHz}$, the return loss was better than $20 \mathrm{~dB}$, and the transmission loss was about $0.2 \mathrm{~dB}$. The structure of the E-plane RF probe and the final simulation result are shown in Figure 5.
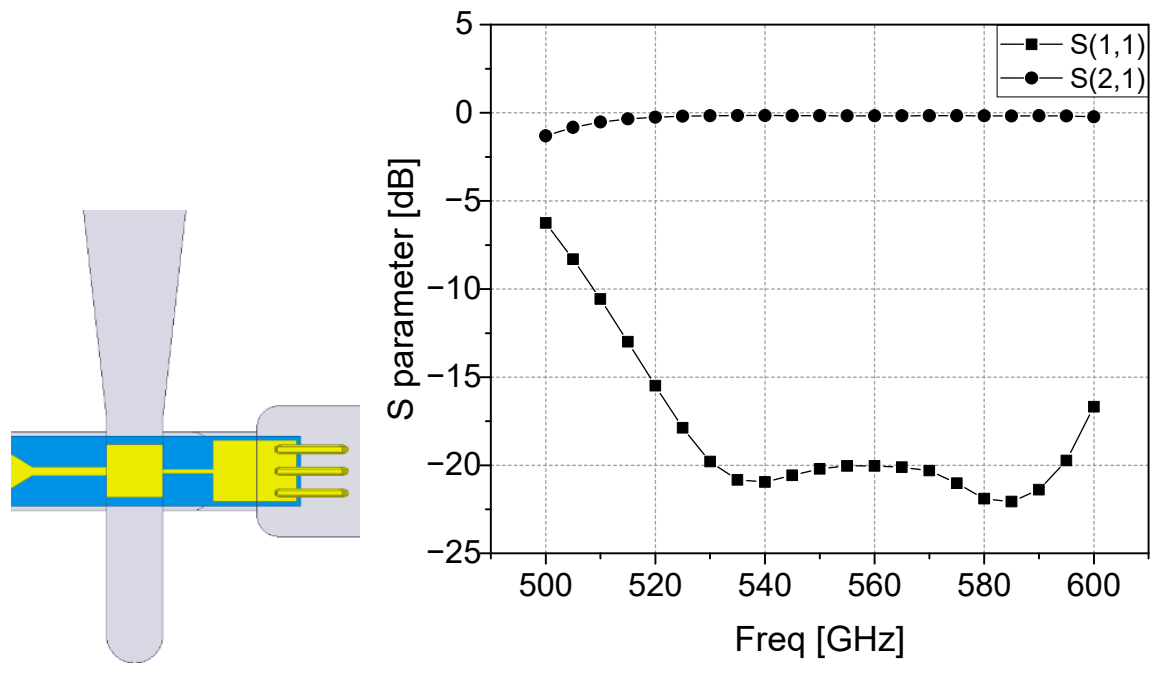

Figure 5. The structure of the E-plane probe and its S-parameter.

After circuit simulation of all passive structures and establishment of the Schottky diode model at the terahertz frequency band, the RF probe and the LO-IF duplexer were imported to the ADS as the files include $S$ parameters of $N$ ports structures, the transmission lines and the diodes were then connected. The circuit simulation diagram of the $560 \mathrm{GHz}$ sub-harmonic mixer is shown in Figure 6. Figure 7 shows the relationship between conversion loss and the input LO power. As the LO power increases, the conversion loss decreases and reaches a certain value. When the LO power is higher than a fixed value, such as $3 \mathrm{dBm}$, the anti-parallel diodes are driven by LO power and the terahertz sub-harmonic mixer with anti-parallel diodes is in the best working condition with the matched impedances of RF and LO ports. When the mixer was pumped with a LO power of $3 \mathrm{~mW}$ and a RF power of $-10 \mathrm{dBm}$ at $555 \mathrm{GHz}$, the spectrum response of the IF output 
is shown in Figure 8. Among these frequency components, the IF is the strongest output signal, and the power of LO, RF and various harmonics are much lower than that of IF. This indicates a good conversion performance of the sub-harmonic mixer.

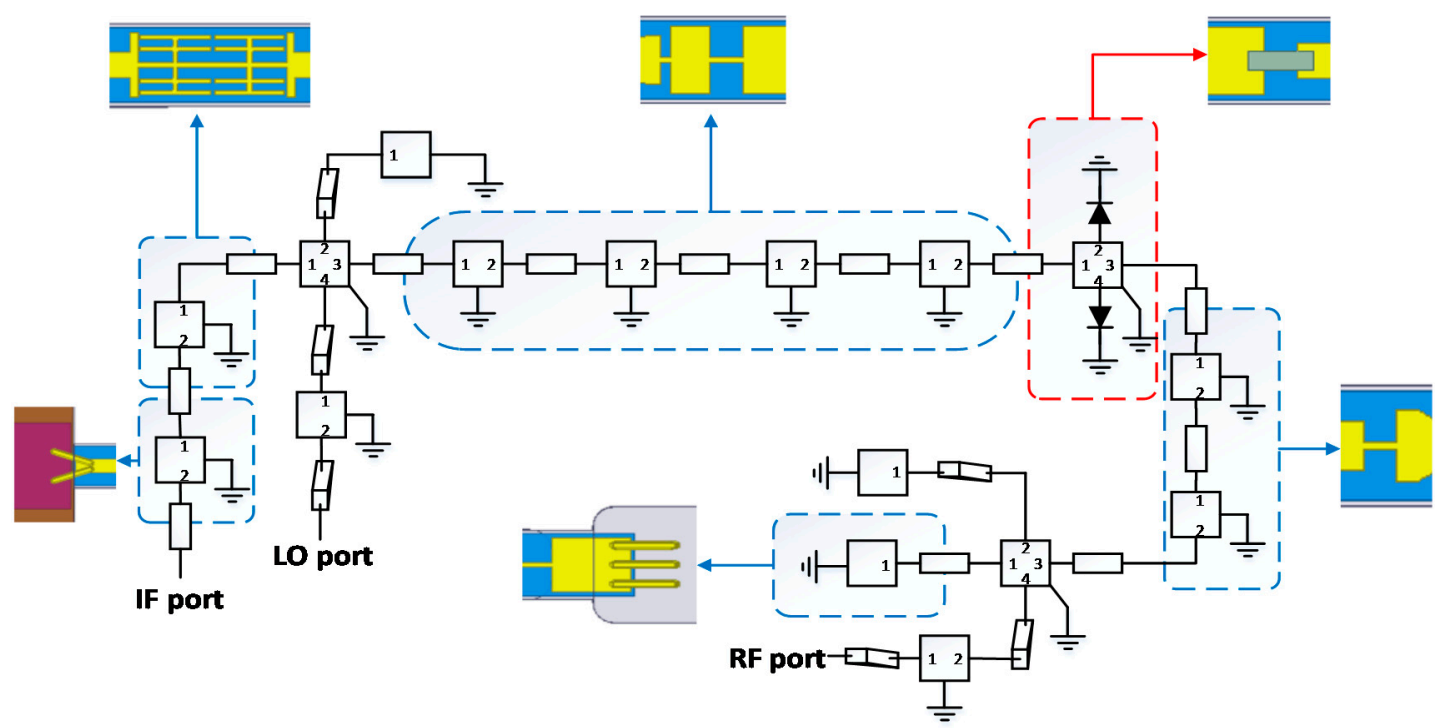

Figure 6. The circuit simulation schematic in the ADS.

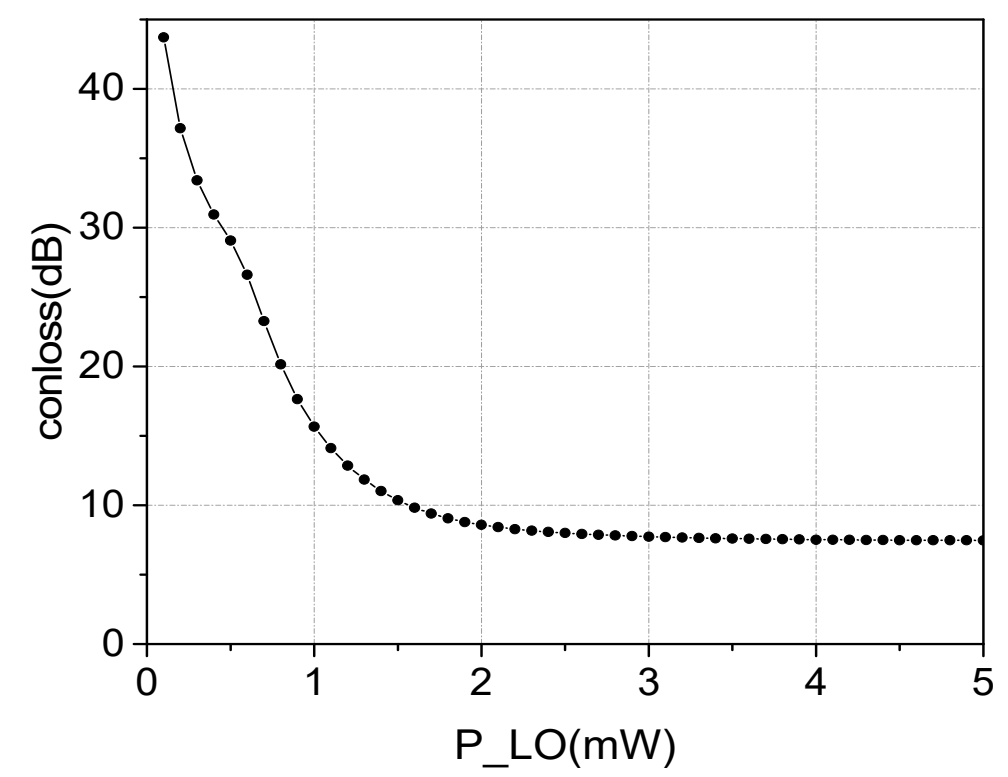

Figure 7. Conversion loss versus the input power of local oscillator. 


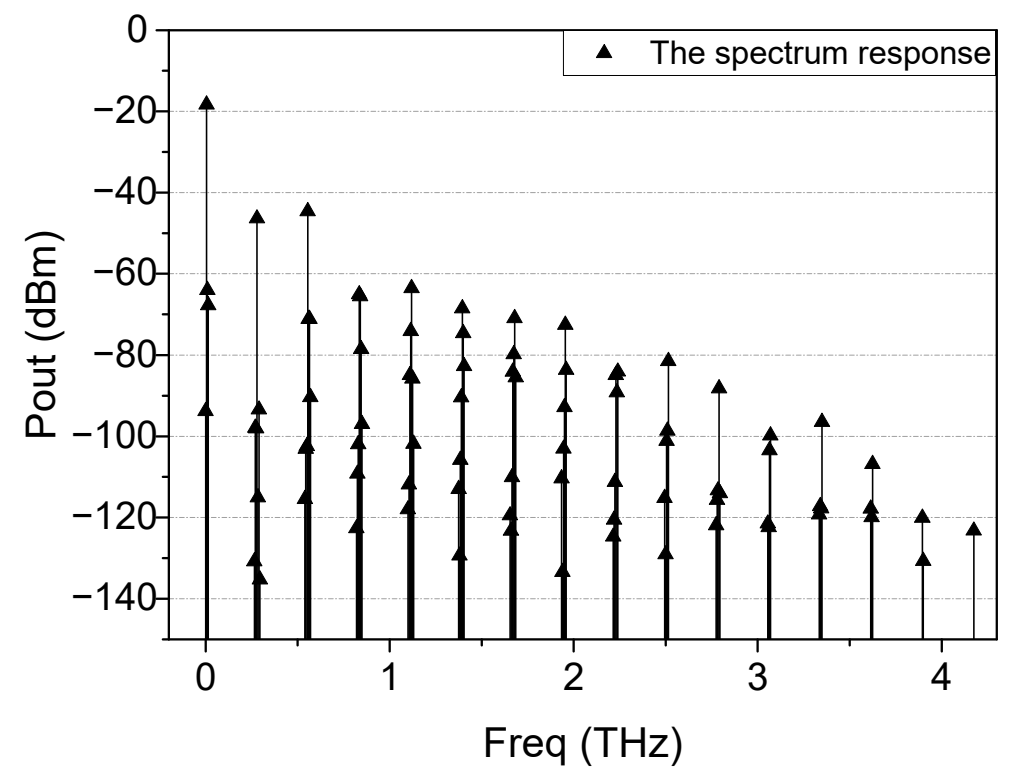

Figure 8. The spectrum response of IF output.

\section{Fabrication and Measurement}

The complete circuit inside the waveguide block is shown in Figure 9. The metal block was fabricated using brass, and electroplated with 2-um-thick gold. The metal block is divided into two parts for processing and assembly. Pins and screws are used to keep these two parts tightly connected. The dimension of the metal block is $23.5 \mathrm{~mm} \times 19 \mathrm{~mm} \times 25 \mathrm{~mm}$. The overall length of the SMS line was reduced to $1.1 \mathrm{~mm}$. During the assembly process, the alignment of the substrate and the cavity is important, and the position of diodes should be accurately fixed. The temperature during the sintering of the substrate and the diode should be controlled to avoid substrate bending during the sintering process. The assembled module of the mixer is shown in Figure 10.

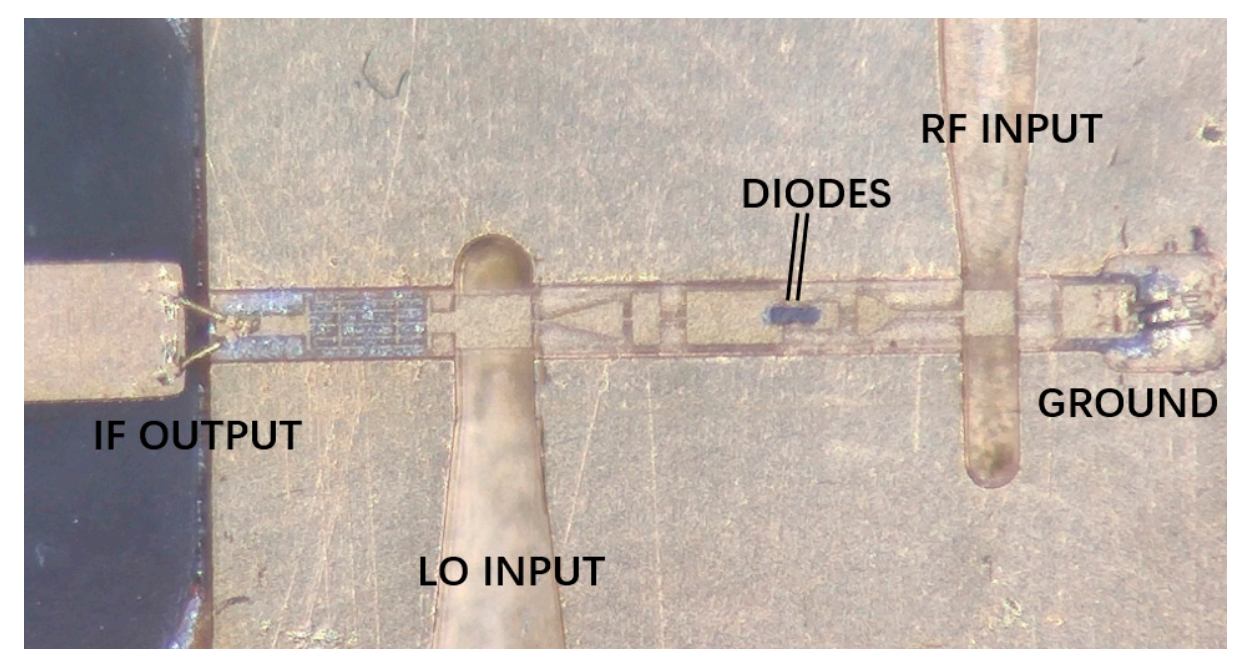

Figure 9. The photo of the sub-harmonic mixer mounted in block. 


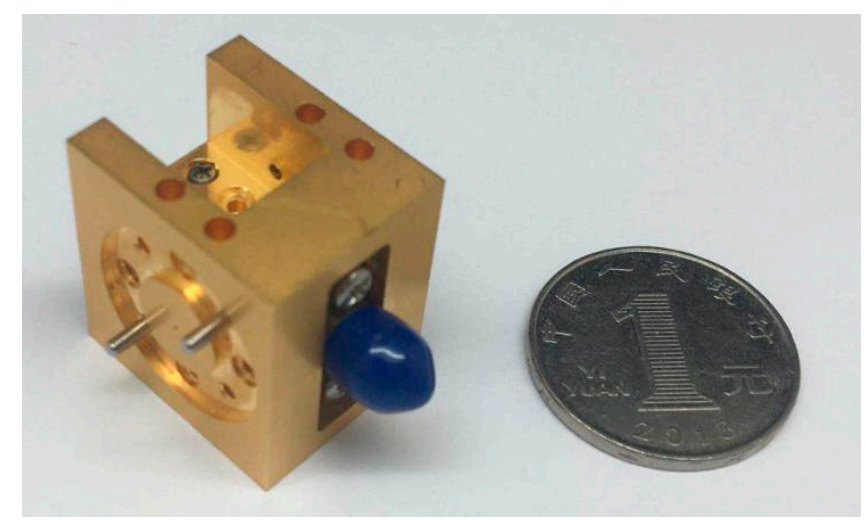

Figure 10. The photo of the assembled sub-harmonic mixer.

During the test, the mixer was characterized in up-conversion for the absence of RF source; the IF ranged from $0.5 \mathrm{GHz}$ to $20 \mathrm{GHz}$ with a power of $-5 \mathrm{dBm}$. An Erickson PM4 power meter was used to measure the output power of the RF port. The mixer was pumped with a LO power of $3 \mathrm{~mW}$ at $280 \mathrm{GHz}$. The LO source was realized by the cascade of a W-band frequency source, a power amplifier, and a frequency tripler. The test platform of the sub-harmonic mixer is shown in Figure 11. When the input power of IF is $-5 \mathrm{dBm}$, the measured double sideband (DSB) conversion loss was about $8 \mathrm{~dB}$. An additional loss of $2.5 \mathrm{~dB}$ was caused in the process of processing, assembly, and testing. The comparison of the simulated and the measured results is shown in Figure 12. A comprehensive comparison of the designed sub-harmonic mixer in the literature is given in Table 1. Compared with other similar designs, this sub-harmonic mixer designed using the HGDM has lower conversion loss, which means better performance. The low LO power reduces the requirements of the $\mathrm{LO}$ source. The combination of low conversion loss and low $\mathrm{LO}$ power makes this sub-harmonic mixer versatile.

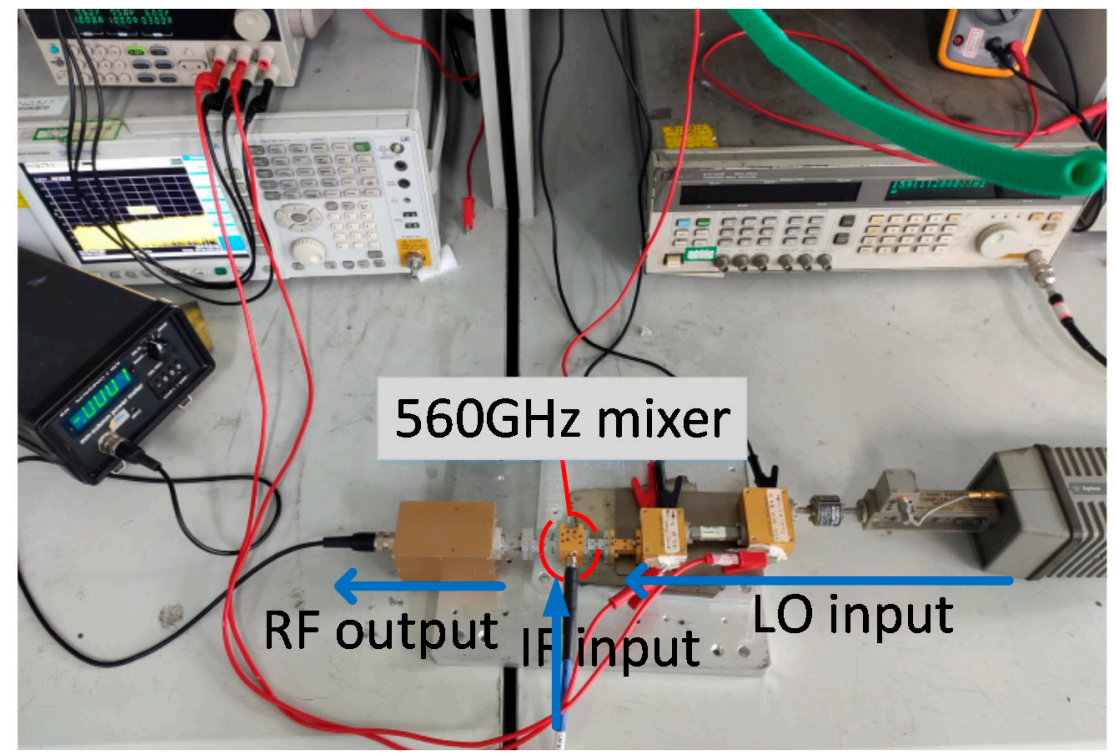

Figure 11. The test platform of the sub-harmonic mixer. 


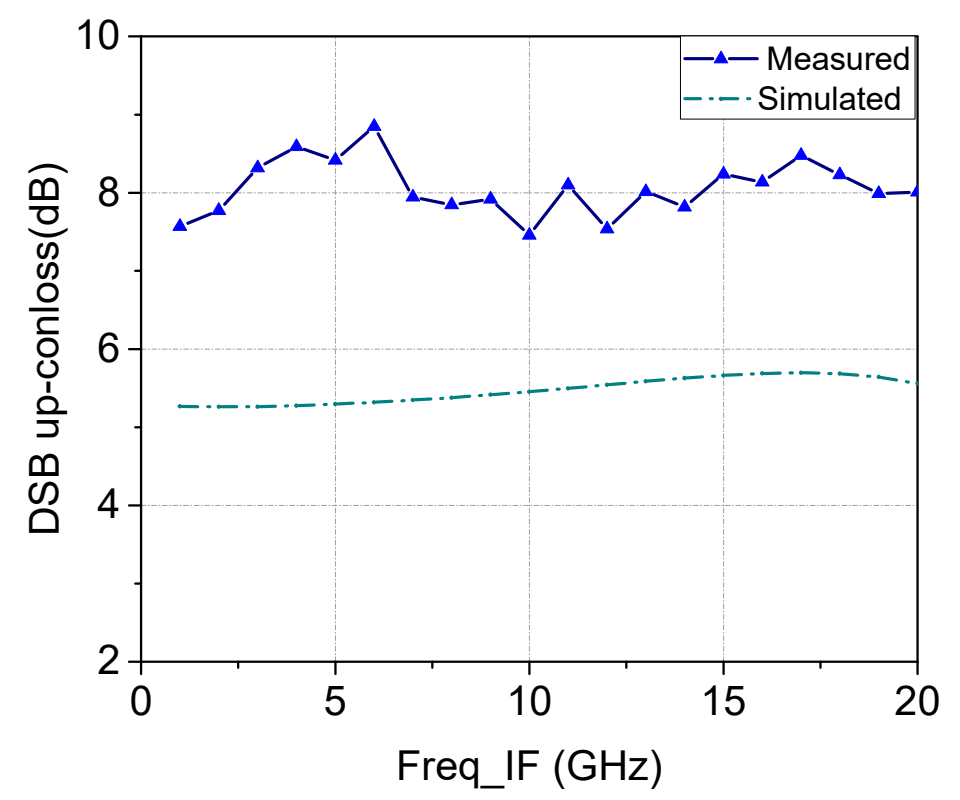

Figure 12. The up-conversion performance of the mixer.

Table 1. Comprehensive comparison of the reported sub-harmonic mixer.

\begin{tabular}{ccccc}
\hline Ref. & $\begin{array}{c}\text { Frequency } \\
\mathbf{( G H z )}\end{array}$ & $\begin{array}{c}\text { Integration } \\
\text { Type }\end{array}$ & LO Power (mW) & $\begin{array}{c}\text { DSB Conversion } \\
\text { Loss (dB) }\end{array}$ \\
\hline$[2]$ & $520-600$ & hybrid & $30-50$ & 7.7 \\
{$[23]$} & $520-590$ & hybrid & $6-10$ & 12 \\
{$[24]$} & $630-720$ & hybrid & $2-8$ & 10.5 \\
{$[25]$} & $530-590$ & monolithic & 1.5 & 9 \\
{$[26]$} & $520-620$ & hybrid & 3 & 5.7 \\
this work & $540-580$ & hybrid & 3 & 8 \\
\hline
\end{tabular}

\section{Conclusions}

In this paper, the design, measurement, and analysis of a $560 \mathrm{GHz}$ sub-harmonic mixer using a novel half-global design method is presented. The mixer using the HGDM achieves average DSB conversion loss of $8 \mathrm{~dB}$ within $565-585 \mathrm{GHz}$. This means that it has a comparable performance to similar designs, such as the products of Virginia Diode, Inc. (VDI). The good test results verify the feasibility of the design method at THz frequencies. This method is a good candidate for a terahertz mixer design.

Author Contributions: Data curation, L.P. and Y.L.; Investigation, J.C.; Resources, R.X. and B.Y.; Writing-review and editing, Y.Z.; Writing-original draft, B.Z. All authors have read and agreed to the published version of the manuscript.

Funding: This research received no external funding.

Conflicts of Interest: The authors declare no conflict of interest.

\section{References}

1. Siegel, P.H. Terahertz technology. IEEE Trans. Microw. Theory Tech. 2002, 50, 910-928. [CrossRef]

2. Thomas, B.; Gill, J.; Maestrini, A.; Lee, C.; Lin, R.; Sin, S.; Peralta, A.; Mehdi, I. An integrated 520-600 GHz GHz sub-harmonic mixer and tripler combination based on GaAs MMIC membrane planar Schottky diodes. In Proceedings of the 35th International Conference on Infrared, Millimeter, and Terahertz Waves, Rome, Italy, 5-10 September 2010; pp. 1-2.

3. Eissa, M.H.; Awny, A.; Ko, M.; Schmalz, K.; Elkhouly, M.; Malignaggi, A.; Ulusoy, A.C.; Kissinger, D. A 220-275 GHz directconversion receiver in 130-nm SiGe:C BiCMOS technology. IEEE Microw. Wirel. Compon. Lett. 2017, 27, 675-677. [CrossRef]

4. Shivan, T.; Weimann, N.; Hossain, M.; Stoppel, D.; Boppel, S.; Ostinelli, O.; Doerner, R.; Bolognesi, C.R.; Krozer, V.; Heinrich, W. A highly efficient ultrawideband traveling-wave amplifier in InP DHBT technology. IEEE Microw. Wirel. Compon. Lett. 2018, 28, 1029-1031. [CrossRef] 
5. Yoon, D.; Kim, J.; Yun, J.; Kaynak, M.; Tillack, B.; Rieh, J.-S. 300-GHz Direct and Heterodyne Active Imagers Based on 0.13- 4 m SiGe HBT Technology. IEEE Trans. Terahertz Sci. Technol. 2017, 7, 536-545. [CrossRef]

6. Kim, J.; Yoon, D.; Yun, J.; Song, K.; Kaynak, M.; Tillack, B.; Rieh, J.-S. Three-dimensional terahertz tomography with transistorbased signal source and detector circuits operating near $300 \mathrm{GHz}$. IEEE Trans. Terahertz Sci. Technol. 2018, 8, 482-491. [CrossRef]

7. Schultz, J.; Herald, D.; Xu, H.; Liu, L.; Bass, R.; Weikle, R.; Lichtenberger, A. The Design, Fabrication and Test Results of a 1.6 THz Superconducting Hot Electron Bolometer Mixer on SOI. IEEE Trans. Appl. Supercond. 2009, 19, 297-300. [CrossRef]

8. Montazeri, S.; Grimes, P.K.; Tong, C.-Y.E.; Bardin, J.C.; Tong, C.E. A 220-GHz SIS Mixer Tightly Integrated with a Sub-HundredMicrowatt SiGe IF Amplifier. IEEE Trans. Terahertz Sci. Technol. 2015, 6, 133-140. [CrossRef]

9. di Gaspare, A.; Giliberti, V.; Casini, R.; Giovine, E.; Evangelisti, F.; Ortolani, M. Sub-terahertz mixer based on heterostructure field effect transistor with integrated antennas. In Proceedings of the 2013 7th European Conference on Antennas and Propagation, Gothenburg, Sweden, 8-12 April 2013; pp. 1736-1739.

10. Pfeiffer, U.R.; Mishra, C.; Rassel, R.M.; Pinkett, S.; Reynolds, S.K. Schottky barrier diode circuits in silicon for future millimeterwave and terahertz applications. IEEE Trans. Microw. Theory Tech. 2008, 56, 364-371. [CrossRef]

11. Redo-Sanchez, A.; Zhang, X.C. Terahertz science and technology trends. IEEE J. Sel. Top. Quantum Electron. 2008, 14, 260-269. [CrossRef]

12. Tonouchi, M. Prospect of terahertz technology. In Proceedings of the International Conference on Applied Electromagnetics and Communications, Dubrovnik, Croatia, 24-26 September 2007; pp. 1-4.

13. Liu, C.; Li, Q.; Xiong, Y.Z. A $340 \mathrm{GHz}$ MMIC $4 \times$ sub-harmonic mixer using silicon-based Schottky barrier diodes. In Proceedings of the 2015 IEEE International Wireless Symposium (IWS 2015), Shenzhen, China, 30 March-1 April 2015; pp. 1-4. [CrossRef]

14. Michaelsen, R.S.; Johansen, T.K.; Krozer, V. Design of a $\times 4$ subharmonic sub-millimeter wave diode mixer, based on an analytic expression for small-signal conversion admittance parameters. In Proceedings of the 2013 SBMO/IEEE MTT-S International Microwave \& Optoelectronics Conference (IMOC), Rio de Janeiro, Brazil, 4-7 August 2013; pp. 1-4. [CrossRef]

15. Cohn, M.; Degenford, J.E.; Newman, B.A. Harmonic Mixing with an Anti-Parallel Diode Pair. In Proceedings of the S-MTT International Microwave Symposium Digest, Atlanta, GA, USA, 12-14 June 1974; pp. 171-172. [CrossRef]

16. Gaojian, L.; Jun, L.; Hui, X.; Xiaoyang, Z.; Shuantao, L.; Hongxi, Y. Design of a 220 GHz subharmonic mixer based on plannar Schottky diode. In Proceedings of the 2017 IEEE Asia Pacific Microwave Conference (APMC), Kuala Lumpar, Malaysia, 13-16 November 2017; pp. 418-421.

17. Mehdi, I.; Marazita, S.; Humphrey, D.; Lee, T.-H.; Dengler, R.; Oswald, J.; Pease, A.; Martin, S.; Bishop, W.; Crowe, T.; et al. Improved 240-GHz subharmonically pumped planar Schottky diode mixers for space-borne applications. IEEE Trans. Microw. Theory Tech. 1998, 46, 2036-2042. [CrossRef]

18. Cui, J.; Zhang, Y.; Xia, D.; Xu, Y.; Xiao, F.; Yan, B.; Xu, R. A 220 GHz broadband sub-harmonic mixer based on global design method. IEEE Access 2019, 7, 30067-30078. [CrossRef]

19. Cui, J.; Zhang, Y.; Xu, Y.; Ye, L.; Xiao, F.; Yan, B.; Xu, R. A 200-240 GHz Sub-Harmonic Mixer Based on Half-Subdivision and Half-Global Design Method. IEEE Access 2020, 8, 33461-33470. [CrossRef]

20. Zhong, F.; Zhang, B.; Fan, Y.; Zhao, M.; Yang, X. A Broadband W-band Subharmonic Mixers Circuit Based on Planar Schottky Diodes. In Proceedings of the 2012 International Conference on Industrial Control and Electronics Engineering, Xi'an, China, 23-25 August 2012; pp. 792-794. [CrossRef]

21. Hu, N.; Xie, W.; Liu, J.; Liu, S.; Zeng, Q.; Luo, Y. Modeling of GaAs Multiplier Schottky Diode in Terahertz Band. In Proceedings of the 2019 IEEE International Conference on Computational Electromagnetics (ICCEM), Shanghai, China, 20-22 March 2019; pp. 1-3. [CrossRef]

22. Wu, C.; Zhang, Y.; Xu, Y.; Bo, Y.; Xu, R. Dual Lumped Ports Technique and Its Applications in Modeling of Planar Schottky Diode in THz Band. IEEE Access 2020, 8, 38001-38009. [CrossRef]

23. Schlecht, E.; Gill, J.; Dengler, R.; Lin, R.; Tsang, R.; Mehdi, I. A Unique 520-590 GHz Biased Subharmonically-Pumped Schottky Mixer. IEEE Microw. Wirel. Compon. Lett. 2007, 17, 879-881. [CrossRef]

24. He, Y.; Tian, Y.; Miao, L.; Jiang, J.; Deng, X. A broadband 630-720 GHz Schottky based sub-harmonic mixer using intrinsic resonances of hammer-head filter. China Commun. 2019, 16, 76-84.

25. Zhao, H.; Drakinskiy, V.; Sobis, P.; Hanning, J.; Bryllert, T.; Tang, A.Y.; Stake, J. Development of a 557 GHz GaAs monolithic membrane-diode mixer. In Proceedings of the 2012 International Conference on Indium Phosphide and Related Materials, Santa Barbara, CA, USA, 27-30 August 2012; pp. 102-105. [CrossRef]

26. Treuttel, J.; Gatilova, L.; Maestrini, A.; Moro-Melgar, D.; Yang, F.; Tamazouzt, F.; Vacelet, T.; Jin, Y.; Cavanna, A.; Mateos, J.; et al. A 520-620-GHz Schottky Receiver Front-End for Planetary Science and Remote Sensing With 1070 K-1500 K DSB Noise Temperature at Room Temperature. IEEE Trans. Terahertz Sci. Technol. 2016, 6, 148-155. [CrossRef] 